QUARTERLY OF APPLIED MATHEMATICS

VOLUME LXVIII, NUMBER 4

DECEMBER 2010, PAGES 783-802

S 0033-569X(2010)01201-X

Article electronically published on September 23, 2010

\title{
DYNAMIC SOCIAL NETWORK MODELS INCORPORATING STOCHASTICITY AND DELAYS
}

\author{
BY \\ H. T. BANKS (Center for Research in Scientific Computation, Center for Quantitative Studies in \\ Biomedicine, North Carolina State University, Raleigh, North Carolina 27695-8212), \\ KERI REHM (Center for Research in Scientific Computation, Center for Quantitative Studies in \\ Biomedicine, North Carolina State University, Raleigh, North Carolina 27695-8212), \\ AND \\ KARYN L. SUTTON (Center for Research in Scientific Computation, Center for Quantitative \\ Studies in Biomedicine, North Carolina State University, Raleigh, North Carolina 27695-8212)
}

\begin{abstract}
Networks are typically studied via computational models, and often investigations are restricted to the static case. Here we extend the work in Banks, Karr, Nguyen and Samuels (2008), which demonstrated a simple dynamical system framework in which to study social network behavior, to include a discrete delay. This delay represents the time lag that is likely required for an agent to change his/her own characteristics (e.g., opinions, viewpoints or behavior) after interacting with an agent possessing different characteristics. Thus this modification adds significantly to the relevance of the model in many potential applications. We have shown that the delays can be incorporated into a stochastic differential equations (SDE) framework in an efficient and computationally tractable way. Through numerical studies, we see novel outcomes when stochasticity, delay, or both are considered, demonstrating the need to include these features should they be present in the network application.
\end{abstract}

1. Introduction. The study of social networks and many other applications of networks have typically been via computational representations of static nodal structures connected by edges defined by pairwise relationships. We refer the reader to [14, 25, 26] for an overview of some current approaches and topics in quantitative studies of social networks. The model developed in [8] provided an alternative simple framework which allows for a dynamic network while generating some of the behavior observed by more complex approaches. The model describes the time evolution of agent's characteristics (e.g., "sociability quotient" and "outlook on life") based on their interactions with agents

Received July 18, 2009.

2000 Mathematics Subject Classification. Primary 91D30, 91C20, 34F05, 34K50.

Key words and phrases. Social networks, stochastic differential equations, delay differential equations, clustering. 
with whom they are connected. Computational results with this model reveal rich dynamics and also reveal the ease with which the model can be extended to enhance its representation of a social network. The results of these efforts suggest that other dynamical systems formulations may be profitably used to study network behavior, including partial differential equations to study spatial effects or delay differential equations for applications in which time lags in communication-based influences on characteristic changes may be important.

We focus here on time delays in stochastic models, which may significantly enhance the relevance of the model to an actual social network. This is done by allowing for a lag in the change in an agent's characteristic(s) based on interactions with agents of different characteristics. Particularly in the context of an individual's viewpoint or opinion on matters of religion, politics, or social issues (alcohol use, abortion, etc.), it is reasonable to assume that these viewpoints are not affected instantaneously upon encountering an individual with a differing viewpoint. While time delays have a direct reasonable interpretation in the context of social networks, they are also significant in a wide variety of other networks, such as information systems, food production networks, biochemical and neural networks, etc. As evidenced by the literature on these types of questions in the applications (for example, 12, 13, 20, 24), there is a general interest in the numerical solution of network models incorporating both stochastic effects and time delays.

The question of whether delays (even if small) make a difference in modeling of physical systems has been of keen interest to mathematical investigators worldwide for some time (see, for example, the references in [2, including extensive efforts in the Russian literature cited therein). It has been known for many years in some applications (antirolling stabilization systems in ships [6, 21, 22, 23, automatic steering of high-velocity aircraft [7, 23], active control in unsteady aeronautics [1, 7], as well as respiratory models with systemic transport delays [3]), that even small delays can be extremely important. Essentially, addition of delays to an $n$-dimensional linear vector system of differential equations (with corresponding $n$ natural frequencies) results in an infinite-dimensional system with an infinite number of natural frequencies [11, 18, so that serious questions arise with respect to concomitant changes in stability, observability, identifiability and controllability as well as qualitative and numerical properties. On the other hand, it is also known [15 that some delays can be "harmless" with respect to properties of importance. Thus, if delays are naturally present (as in some social networks) in a problem, the question arises as to whether one can safely ignore these in models when performing computations, carrying out qualitative investigations, designing control regimes, etc. In this paper we focus on qualitative changes that arise when including delays in stochastic social network models such as those developed in 8 . In particular, we seek to determine, through computational studies, whether delays should be given consideration in these types of models, or if they can be neglected when investigating clustering and other model behaviors. Further, we seek to understand what (if any) magnitude of delay might impact the observed system dynamics.

The model developed in [8] does not correspond to a continuous diffusion process, and hence the usual Markov process methodologies cannot be employed directly on that 
model. For example, conversion of stochastic differential equations (SDEs) to an equivalent Fokker-Planck (FP) system, a partial differential equation of the transition probabilities for the corresponding Markov process of the SDEs, can only be done after a modest but nontrivial change in the formulation of $[8$. This is discussed in 10 . Numerical implementation of nonlinear time delays into a system of SDEs of large dimension could potentially be problematic. In the technical report [10, we modify the model of 8], demonstrate that the modification does not alter in significant ways the qualitative behavior of solutions, and consider the model in both SDE and FP formulations to determine which to pursue as a convenient means to study network dynamics with delays. Through this exploration it was evident that the Fokker-Planck formulation is not as amenable as the system of SDEs to considering inclusion of delays for the underlying social network model. Thus, we study here the extended version of the model derived in [10, in which the coefficients have been modified so that the corresponding SDE does describe a continuous Markov diffusion process. In this paper, we explore the effect of a discrete time delay on the clustering behavior of the dynamic social network model. (We remark that one could also readily include distributed delays using ideas such as those in [5] along with those presented below.) We first study the isolated effect of introducing the time delay into the deterministic version of the model obtained by suppressing noise in the system. We expand upon the findings in 8 to include stochastic realizations carried out for a wider range of parameter values in which distinct classes of behavior occur. Finally, the effects of stochasticity and delay are included simultaneously, and the significance of including both of these effects in these types of models is discussed.

2. Model. We discuss a model of a social network as a system of stochastic differential equations (SDEs) slightly modified from a previously introduced version in 8] so that the SDE model represents a classic diffusion process. The model describes the dynamics of agent characteristics $C_{i}(t) \in K \subset \mathbb{R}^{m}$, where $m$ is the number of characteristics and $K$ is a (compact) constraint set of values of the characteristics of each agent $i$, $i \in \mathcal{N}=\{1, \ldots, N\}$. The characteristics of each agent $i$ are affected by the characteristics of other agents $i^{\prime}$ to which they are connected. The strength of the connection from agent $i$ to agent $i^{\prime}$ at time $t$ is represented by the connectivity $e\left(i, i^{\prime}, t\right)$. These connectivities are not necessarily symmetric, so it is possible that $e\left(i, i^{\prime}, t\right) \neq e\left(i^{\prime}, i, t\right)$. A connection from agent $i$ to agent $i^{\prime}$ 'exists' at time $t$ if and only if $e\left(i, i^{\prime}, t\right)>0$. When a connection between two agents exists, the relationship between the two agents is assumed to be homophilic, and when a connection between two agents does not exist, the characteristics of agent $i^{\prime}$ do not affect those of agent $i$. The modified model dynamics are given by

$$
\begin{aligned}
& d C_{i}(t)=\frac{\beta_{i}}{\sum_{i^{\prime} \neq i} \phi_{i, i^{\prime}}(t)} \sum_{i^{\prime} \neq i} \phi_{i, i^{\prime}}(t)\left[C_{i^{\prime}}(t)-C_{i}(t)\right] d t+\sigma d W_{i}^{C}(t), \\
& d e\left(i, i^{\prime}, t\right)=f\left(\left\|C_{i}(t)-C_{i^{\prime}}(t)\right\|^{2}\right) d t+\gamma d W_{i, i^{\prime}}^{e}(t),
\end{aligned}
$$

where $\beta_{i}>0$ is an agent-specific parameter determining the extent to which agent $i$ 's characteristics are affected by other agents. We define $\phi_{i, i^{\prime}}(t)=\frac{1}{2}\left(e\left(i, i^{\prime}, t\right)+\left|e\left(i, i^{\prime}, t\right)\right|\right)$ so that no agent $i^{\prime}$ affects agent $i$ if $e\left(i, i^{\prime}, t\right) \leq 0$. In this model, the characteristics of each agent change according to a weighted average difference between their characteristics 
and those of other agents to which they are connected. The difference is weighted by $\phi_{i, i^{\prime}}(t)$ so that the characteristics of an agent $i$ are more strongly influenced by those of agents with whom agent $i$ is more strongly connected. We set $e(i, i, t)=0$ for all $i$ and $t$ to avoid agents influencing their own characteristics, and it is assumed that $\frac{\beta_{i}}{\sum_{i^{\prime} \neq i} \phi_{i, i^{\prime}}(t)}=0$ if $\sum_{i^{\prime} \neq i} \phi_{i, i^{\prime}}(t)=0$ to maintain definition of the model if a single-agent cluster is formed. In accordance with the work done in $\left[8\right.$, we take $f(\xi)=2 e^{-b \xi}-1$ where $b>0$ is a constant and $\|\cdot\|$ denotes the usual Euclidean norm in $\mathbb{R}^{m}$. Changes in characteristics and connectivities are subject to uncertainty via the Wiener processes $\sigma W_{i}^{C}(\cdot)$ and $\gamma W_{i, i^{\prime}}^{e}(t)$ with variances $\sigma$ and $\gamma$, respectively.

The version of the model used here therefore represents a classic diffusion process and has an equivalent Fokker-Planck representation, discussed in more detail in [10]. Although we did not choose to pursue further analysis of the Fokker-Planck system, we continue studying this version of the model, as no qualitative differences were observed in model dynamics between the version introduced in [8] and this version.

In an effort to add to the relevance of the model, we incorporate a discrete delay into Equation (11). The dynamics of the characteristics are now given by

$$
d C_{i}(t)=\frac{\beta_{i}}{\sum_{i^{\prime} \neq i} \phi_{i, i^{\prime}}(t-\tau)} \sum_{i^{\prime} \neq i} \phi_{i, i^{\prime}}(t-\tau)\left[C_{i^{\prime}}(t-\tau)-C_{i}(t-\tau)\right] d t+\sigma d W_{i}^{C}(t) .
$$

This modification represents the time delay likely required for an agent to actually be influenced by another agent having different characteristics. That is, a change in an agent's characteristics occurs $\tau$ time units after interacting with agents of different characteristics. One way to think of this would be to consider the characteristics as being opinions or viewpoints, and it then is reasonable to represent a change in one's opinion as not occurring instantaneously after exposure to a different viewpoint. Connections between agents are still determined by the characteristics at the current time $t$, and Equation (2) is unchanged.

3. Numerical methods. We outline here the numerical methods used to compute solutions to the model (2)-(3). First we discuss the typical fourth-order Runge-Kutta scheme for stochastic systems in Section 3.1. This method is for the non-delayed version of the model $(\tau=0)$, and was also used in [8]. The implementation of a delay in a (deterministic) system of nonlinear differential equations with the fourth-order RungeKutta method is contained in Section 3.2. Then the computation of the SDE model with delays is a matter of modifying the RK in Section 3.2 to incorporate stochasticity as in Section 3.1

3.1. Stochastic Runge-Kutta. We describe how to numerically implement the stochastic differential equation model (11)-(2) using the stochastic analogue of the fourth-order Runge-Kutta method. We use a constant step size $h=\Delta t_{n}=t_{n}-t_{n-1}$ in the interval $\left[t_{0}, T\right]$ where $t_{0}=0$ and the final time $T$ is always reported for the solutions discussed in Section 4 
The connections between agents are approximated by

$$
\begin{aligned}
e\left(i, i^{\prime}, t_{n}\right)= & e\left(i, i^{\prime}, t_{n-1}\right)+p_{0} f\left(\left\|C_{i}\left(t_{n-1}\right)-C_{i^{\prime}}\left(t_{n-1}\right)\right\|^{2}\right) h \\
& +\left(p_{1}+p_{2}\right) f\left(\left\|C_{i}\left(t_{n-1}+\frac{1}{2} h\right)-C_{i^{\prime}}\left(t_{n-1}+\frac{1}{2} h\right)\right\|^{2}\right) h \\
& +p_{3} f\left(\left\|C_{i}\left(t_{n}\right)-C_{i^{\prime}}\left(t_{n}\right)\right\|^{2}\right) h+\gamma \Delta W_{i, i^{\prime}}^{e}\left(t_{n}\right),
\end{aligned}
$$

where $f$ is from the right side of (2), $p_{0}=p_{3}=\frac{1}{6}, p_{1}=p_{2}=\frac{1}{3}$, and $\Delta W_{i, i^{\prime}}^{e}\left(t_{n}\right)=$ $W_{i, i^{\prime}}^{e}\left(t_{n}\right)-W_{i, i^{\prime}}^{e}\left(t_{n-1}\right)$ are standard Wiener increments $([17$, p. 489). These are generated by sampling from a random variable with mean zero and variance $h=\Delta t_{n}$. We assume that the value of $C_{i}\left(t_{n}+\frac{1}{2} h\right)$ can be approximated linearly from $C_{i}\left(t_{n-1}\right)$ to $C_{i}\left(t_{n}\right)$ when $\Delta t_{n}$ is small, and therefore $C_{i}\left(t_{n-1}+\frac{1}{2} h\right)$ may be approximated by $\frac{C_{i}\left(t_{n-1}\right)+C_{i}\left(t_{n}\right)}{2}$.

Approximating Equation (1) is more involved since its right side depends on $C_{i}(t)$ as well as on the connections $e\left(i, i^{\prime}, t\right)$. If we let

$$
g_{i}\left(t, C_{i}(t)\right)=\frac{\beta_{i}}{\sum_{i^{\prime} \neq i} \phi_{i, i^{\prime}}(t)} \sum_{i^{\prime} \neq i} \phi_{i, i^{\prime}}(t)\left[C_{i^{\prime}}(t)-C_{i}(t)\right],
$$

then Equation (11) is approximated by

$$
C_{i}\left(t_{n}\right)=C_{i}\left(t_{n-1}\right)+p_{0} F_{i 0} h+p_{1} F_{i 1} h+p_{2} F_{i 2} h+p_{3} F_{i 3} h+\sigma \Delta W_{i}^{C} .
$$

Again, $\Delta W_{i}^{C}(t)$ are standard Wiener increments. Necessary quantities to compute (5) are

$$
\begin{aligned}
F_{i 0} & =g_{i}\left(t_{n-1}, C_{i}\left(t_{n-1}\right)\right) \\
C_{i}^{(1)}\left(t_{n-1}\right) & =C_{i}\left(t_{n-1}\right)+\frac{1}{2} F_{i 0} h+\sigma \frac{1}{2} \Delta W_{i}^{C} \\
F_{i 1} & =g_{i}\left(t_{n-1}+\frac{1}{2} h, C_{i}^{(1)}\left(t_{n-1}\right)\right) \\
C_{i}^{(2)}\left(t_{n-1}\right) & =C_{i}\left(t_{n-1}\right)+\frac{1}{2} F_{i 1} h+\sigma \frac{1}{2} \Delta W_{i}^{C} \\
F_{i 2} & =g_{i}\left(t_{n-1}+\frac{1}{2} h, C_{i}^{(2)}\left(t_{n-1}\right)\right) \\
C_{i}^{(3)}\left(t_{n-1}\right) & =C_{i}\left(t_{n-1}\right)+F_{i 2} h+\sigma \Delta W_{i}^{C} \\
F_{i 3} & =g_{i}\left(t_{n-1}+h, C_{i}^{(3)}\left(t_{n-1}\right)\right),
\end{aligned}
$$

where the constants $p_{0}, p_{1}, p_{2}, p_{3}$ take on the same values as in (44). The constraint $C_{i}\left(t_{n}\right) \in K$ is imposed by reassigning an appropriate value on the boundary of $K$ to a characteristic $C_{i}\left(t_{n}\right)$ if it is ever assigned a value outside the set $K$.

3.2. Runge-Kutta with nonlinear delay. We next outline a linear spline approximation to the system (2)-(3), which we consider first in the deterministic case $(\sigma=\gamma=0)$. Banks and Kappel developed general spline approximation methods for systems of differential equations in which all delay terms are linear in 9 . This work was extended in [4] to address the possibility of nonlinear terms involving a delay.

Consider the system

$$
\dot{x}(t)=\mathcal{F}\left(x(t), x_{t}, x\left(t-\tau_{1}\right), \ldots, x\left(t-\tau_{\nu}\right)\right)+\mathcal{G}(t)
$$


for $0 \leq t \leq T, x_{0}=\phi, x(0)=\eta$ where $\mathcal{F}$ is a nonlinear function, $\mathcal{F}=\mathcal{F}\left(\eta, \phi, y_{1}, \ldots, y_{\nu}\right)$, which maps $Z \times \mathbb{R}^{n \nu} \rightarrow \mathbb{R}^{n}$. The state space $Z$ here is $Z=\mathbb{R}^{n} \times L_{2}\left(-r, 0 ; \mathbb{R}^{n}\right)$ where $0<\tau_{1}<\cdots<\tau_{v}=r$. Here $x_{t}$ denotes the usual function $x_{t}=x(t+\theta),-r \leq \theta \leq 0$ and $\phi \in H^{1}(-r, 0)[9$.

Let us define the function $F: \mathbb{R}^{n} \times C\left(-r, 0 ; \mathbb{R}^{n}\right) \subset Z \rightarrow \mathbb{R}^{n}$ as

$$
F(z)=F(\eta, \psi)=\mathcal{F}\left(\eta, \psi, \psi\left(-\tau_{1}\right), \ldots, \psi\left(-\tau_{\nu}\right)\right),
$$

where $\mathcal{F}$ is the function in (6).

The nonlinear operator $\mathcal{A}: D(\mathcal{A}) \subset Z \rightarrow Z$, where $D(\mathcal{A})$ is given by

$$
D(\mathcal{A})=\left\{(\psi(0), \psi) \mid \psi \in H^{1}(-r, 0)\right\},
$$

is defined by

$$
\mathcal{A}(\psi(0), \psi)=(F(\psi(0), \psi), D \psi) .
$$

Let $z(t ; \phi, \mathcal{G})=\left(x(t ; \phi, \mathcal{G}), x_{t}(\phi, \mathcal{G})\right)$, where $x$ is the solution of (6) for $\phi \in H^{1}$ and $\mathcal{G} \in L_{2}$. Then, for initial data $\zeta=(\phi(0), \phi), z(\phi, \mathcal{G})$ is the unique solution on $[0, T]$ of

$$
z(t)=\zeta+\int_{0}^{t}\{\mathcal{A} z(s)+(\mathcal{G}(s), 0)\} d s
$$

The approximation method implemented for our problem is based on the linear spline subspaces $Z^{N}$ approximating the state space $Z$. The scalar linear splines $e_{j}^{N}$ which are used to form the basis functions have nodes at $t_{j}=-j \frac{\tau}{N} j=0,1, \ldots, N$, and are piecewise linear functions defined on $[-\tau, 0]$ such that

$$
e_{j}^{N}\left(t_{i}\right)=\delta_{i j}=\left\{\begin{array}{cc}
1 & \text { if } i=j \\
0 & \text { otherwise }
\end{array} .\right.
$$

The basis functions are $\hat{\beta}^{N}=\left(\beta^{N}(0), \beta^{N}\right)$, where $\beta^{N}=\left(e_{0}^{N}, e_{1}^{N}, \ldots, e_{N}^{N}\right) \otimes I_{n}$, and $I_{n}$ is the $n \times n$ identity matrix. If we let $Z_{1}^{N}$ denote the corresponding linear spline subspace of $Z$, it has dimension $\operatorname{dim} Z_{1}^{N}=k_{n}=n(N+1)$. We seek approximate solutions $z^{N}$ that are elements of $Z_{1}^{N}$ with coordinate vector $\alpha^{N}$, or $z^{N}=\hat{\beta}^{N} \alpha^{N}=\sum_{j=0}^{N}\left(e_{j}^{N}(0), e_{j}^{N}\right) a_{j}^{N}$ where $a_{j}^{N} \in \mathbb{R}^{n}$ such that $\alpha^{N}=\operatorname{col}\left(a_{0}^{N}, \ldots, a_{N}^{N}\right)$.

Let $P^{N}=P_{\tilde{g}}^{N}$ be the orthogonal projection of $Z$ onto $Z^{N}=Z_{1}^{N}$ so that $P^{N} z \rightarrow z$ for all $z \in Z$. The weighted inner product is defined by

$$
\left\langle\left(\eta_{1}, \phi_{1}\right),\left(\eta_{2}, \phi_{2}\right)\right\rangle_{\tilde{g}}=\eta_{1}^{T} \eta_{2}+\int_{-\tau}^{0} \phi_{1}(\theta)^{T} \phi_{2}(\theta) \tilde{g}(\theta) d \theta .
$$

Because we are considering only one discrete delay here, we have that $\tilde{g}(\theta) \equiv 1$ and $m=1$ in [9] and [4]. Then we approximate the operator $\mathcal{A}$ by $\mathcal{A}^{N}=P^{N} \mathcal{A} P^{N}$ and the approximating equations are defined by

$$
z^{N}(t)=P^{N} \zeta+\int_{0}^{t}\left\{\mathcal{A}^{N}\left(z^{N}(s)\right)+P^{N}(\mathcal{G}(s), 0)\right\} d s .
$$

Because $Z_{1}^{N}$ is finite-dimensional, this is equivalent to the ordinary differential equation (ODE) system

$$
\dot{z}^{N}(t)=\mathcal{A}^{N}\left(z^{N}(t)\right)+P^{N}(\mathcal{G}(t), 0), \quad z^{N}(0)=P^{N} \zeta .
$$


The approximate initial condition $P^{N} \zeta=P^{N}(\phi(0), \phi)$ is determined by the orthogonality relationship

$$
\left\{P^{N}(\phi(0), \phi)-(\phi(0), \phi)\right\} \perp Z^{N},
$$

where the $\tilde{g}$ subscript has been suppressed because $\tilde{g}(\theta) \equiv 1$. This is equivalent to writing

$$
\left\langle\hat{\beta}^{N} \alpha^{N}-(\phi(0), \phi), \hat{\beta}^{N}\right\rangle=0 .
$$

By the properties of inner products,

$$
\left\langle\hat{\beta}^{N}, \hat{\beta}^{N}\right\rangle \alpha^{N}-\left\langle(\phi(0), \phi), \hat{\beta}^{N}\right\rangle=0 .
$$

We let $Q^{N}=\left\langle\hat{\beta}^{N}, \hat{\beta}^{N}\right\rangle$ and $h^{N}(\zeta)=\left\langle(\phi(0), \phi), \hat{\beta}^{N}\right\rangle$. By applying the definition of the inner product, we have the following expressions for $Q^{N}$ and $h^{N}$ :

$$
Q^{N}=\beta^{N}(0)^{T} \beta^{N}(0)+\int_{-\tau}^{0} \beta^{N}(\theta)^{T} \beta^{N}(\theta) d \theta
$$

and

$$
h^{N}(\phi(0), \phi)=\beta^{N}(0)^{T} \phi(0)+\int_{-\tau}^{0} \beta^{N}(\theta)^{T} \phi(\theta) d \theta,
$$

where $(\phi(0), \phi)$ are initial data for the system ([6), $x(0)=\phi(0)$ and $x(\theta)=\phi(\theta)$, for $\theta \in[-\tau, 0]$. Note that the dimension of $Q^{N}$ is $\operatorname{dim} Q^{N}=k_{n} \times k_{n}$. In the case of linear spline approximations, $Q^{N}$ is given by

$$
Q^{N}=\frac{\tau}{N}\left[\begin{array}{cccccc}
\frac{N}{\tau}+\frac{1}{3} & \frac{1}{6} & 0 & \cdots & \cdots & 0 \\
\frac{1}{6} & \frac{2}{3} & \frac{1}{6} & 0 & \cdots & \vdots \\
0 & \ddots & \ddots & \ddots & \ddots & \vdots \\
\vdots & \cdots & 0 & \frac{1}{6} & \frac{2}{3} & \frac{1}{6} \\
0 & \cdots & \cdots & 0 & \frac{1}{6} & \frac{1}{3}
\end{array}\right] \otimes I_{n}
$$

Then we can proceed by calculating the coordinate vector $\alpha^{N}$ for the initial piece of the solution by solving the system

$$
Q^{N} \alpha^{N}=h^{N}(\phi(0), \phi) .
$$

We can solve this via Gaussian elimination, but since the dimension of our problem is large, so is the size of $Q^{N}$. Therefore, we use the fact that $Q^{N}$ is a tri-banded matrix to reduce the number of operations performed.

Then $z^{N}(0)=\hat{\beta}^{N} \alpha^{N}=\hat{\beta}^{N} w_{0}^{N}$, which implies $\alpha^{N}=w_{0}^{N}$, and we have the initial condition for the ODE system (7). In solving this system, we need to compute $\mathcal{A}^{N}\left(z^{N}(t)\right)$ at each time $t$ due to the nonlinearity of $\mathcal{A}^{N}$. For each time $t$, the element $z^{N}(t) \in Z^{N}$ can be written as $z^{N}(t)=\hat{\beta}^{N} \alpha^{N}(t)$ for some coordinate vector $\alpha^{N}(t)$. The operator $\mathcal{A}$ applied to $z^{N}(t)$ is $\mathcal{A}\left(z^{N}(t)\right)=\mathcal{A}\left(\beta^{N}(0) \alpha^{N}(t), \beta^{N} \alpha^{N}(t)\right)=$ $\left(F\left(\beta^{N}(0) \alpha^{N}(t), \beta^{N} \alpha^{N}(t)\right), D \beta^{N} \alpha^{N}(t)\right)$. Then

$$
\mathcal{A}^{N}=P^{N} \mathcal{A} P^{N} z=P^{N}\left(F\left(\beta^{N}(0) \alpha^{N}(t), \beta^{N} \alpha^{N}(t)\right), D \beta^{N} \alpha^{N}(t)\right) .
$$


Because $\mathcal{A}^{N}\left(z^{N}(t)\right) \in Z^{N}$, it also has a representation of the form $\mathcal{A}^{N}\left(z^{N}(t)\right)=\hat{\beta}^{N} \gamma^{N}(t)$. Using the orthogonality relation as before, we have

$$
\begin{aligned}
& \left\{\mathcal{A}^{N}\left(\hat{\beta}^{N} \alpha^{N}(t)\right)-\hat{\beta}^{N} \gamma^{N}(t)\right\} \perp Z^{N} \\
& \left\langle\left(\hat{\beta}^{N} \gamma^{N}(t)-\left(F\left(\beta^{N}(0) \alpha^{N}(t), \beta^{N} \alpha^{N}(t)\right), D \beta^{N} \alpha^{N}(t)\right)\right), \hat{\beta}^{N}\right\rangle=0 \\
& \left\langle\hat{\beta}^{N}, \hat{\beta}^{N}\right\rangle \gamma^{N}(t)=\left\langle\hat{\beta}^{N},\left(F\left(\beta^{N}(0) \alpha^{N}(t), \beta^{N} \alpha^{N}(t)\right), D \beta^{N} \alpha^{N}(t)\right)\right\rangle \\
& Q^{N} \gamma^{N}(t)=h^{N}\left(F\left(\beta^{N}(0) \alpha^{N}(t), \beta^{N} \alpha^{N}(t)\right), D \beta^{N} \alpha^{N}(t)\right) .
\end{aligned}
$$

The matrix $Q^{N}$ is unchanged from before. The definition of the inner product is again used to obtain $h^{N}$, which is given by

$$
\begin{array}{r}
h^{N}\left(\mathcal{A} z^{N}(t)\right)=\beta^{N}(0)^{T} F\left(\beta^{N}(0) \alpha^{N}(t), \beta^{N} \alpha^{N}(t)\right) \\
+\int_{-\tau}^{0} \beta^{N}(\theta)^{T} D \beta^{N}(\theta) d \theta \alpha^{N}(t) .
\end{array}
$$

In the above expression the integral $\int_{-\tau}^{0} \beta^{N}(\theta)^{T} D \beta^{N}(\theta) d \theta$ does not depend on time and therefore will only need to be computed once during time stepping. We thus have an equation that one can solve for $\gamma^{N}(t)$, and which can be used in the implementation of an ODE scheme for the system (7).

Here we describe the Runge-Kutta method for one time step of the ODE system (7). This scheme is given by

$$
z^{N}(t+\Delta t)=z^{N}(t)+\frac{1}{6} \Delta t\left(k_{1}+2 k_{2}+2 k_{3}+k_{4}\right) .
$$

The quantities $k_{1}, k_{2}, k_{3}, k_{4}$ are

$$
\begin{aligned}
& k_{1}=\mathcal{A}^{N}\left(z^{N}(t)\right)=\hat{\beta}^{N} \gamma_{1}^{N}(t), \\
& k_{2}=\mathcal{A}^{N}\left(z^{N}(t)+\frac{1}{2} \Delta t k_{1}\right)=\mathcal{A}^{N}\left(\hat{\beta}^{N} \alpha^{N}(t)+\frac{1}{2} \Delta t k_{1}\right)=\hat{\beta}^{N} \gamma_{2}^{N}(t), \\
& k_{3}=\mathcal{A}^{N}\left(z^{N}(t)+\frac{1}{2} \Delta t k_{2}\right)=\mathcal{A}^{N}\left(\hat{\beta}^{N} \alpha^{N}(t)+\frac{1}{2} \Delta t k_{2}\right)=\hat{\beta}^{N} \gamma_{3}^{N}(t), \\
& k_{4}=\mathcal{A}^{N}\left(z^{N}(t)+\Delta t k_{3}\right)=\mathcal{A}^{N}\left(\hat{\beta}^{N} \alpha^{N}(t)+\Delta t k_{3}\right)=\hat{\beta}^{N} \gamma_{4}^{N}(t) .
\end{aligned}
$$

We solve equation (11) for $\gamma_{i}(t)$ to use for $k_{i}$ with $i=1, \ldots, 4$. For example, $\gamma_{1}^{N}$ is obtained by solving $Q^{N} \gamma_{1}^{N}=\bar{h}^{N}$ by Gaussian elimination (again, using the tri-bandedness of $Q^{N}$ to reduce the computations), where $\bar{h}^{N}$ is

$$
\bar{h}^{N}=\beta^{N}(0)^{T} F\left(\beta^{N}(0) \alpha^{N}(t), \beta^{N} \alpha^{N}(t)\right)+\int_{-\tau}^{0} \beta^{N}(\theta)^{T} D \beta^{N}(\theta) d \theta \alpha^{N}(t) .
$$

For $k_{2}, k_{3}, k_{4}$, we solve $Q^{N} \gamma_{i}^{N}=\bar{h}_{i}^{N}$ with $\bar{h}_{i}^{N}$ given by

$$
\bar{h}_{i}^{N}=\beta^{N}(0)^{T} F\left(\beta^{N}(0) \alpha_{i}^{N}(t), \beta^{N} \alpha_{i}^{N}(t)\right)+\int_{-\tau}^{0} \beta^{N}(\theta)^{T} D \beta^{N}(\theta) d \theta \alpha_{i}^{N}(t) .
$$

Here we have used the notation $\hat{\beta}^{N} \alpha_{i}^{N}(t)=\hat{\beta}^{N} \alpha^{N}(t)+\frac{1}{2} \Delta t k_{i-1}$ for $i=2,3$, and $\hat{\beta}^{N} \alpha_{4}^{N}(t)=\hat{\beta}^{N} \alpha^{N}(t)+\Delta t k_{3}$. 
4. Computational results. We first discuss solutions of the deterministic version of the social network model to address whether and to what extent delays have a significant impact on model dynamics. The results of this investigation led us to explore some stochastic scenarios which were not previously explored in [8]. Thus, we include the results for the stochastic model without delay for comparison to the results when the delay is positive. As a result, we are able to distinguish between effects on clustering behavior that are due to the time delay, stochasticity, or a combination of these two factors. We use here the same 10-agent, 2-characteristic example as in [8] (with, of course, the stochastic model modified as in (1)).

While the slightly modified model (11)-(2) from that in [8] exhibit qualitatively similar behavior, parameter values $(b, \sigma, \gamma)$ for which given dynamics are observed differ. The clustering outcomes as a function of the decay parameter $b$ in the function $f$ are:

- 1 cluster of all agents for $b \in[0,0.000155)$,

- 2 clusters: Agents 2 and 7, other agents for $b \in[0.000155,0.01390)$,

- 3 clusters: Agent 2, Agent 7, other agents for $b \in[0.01390, \infty)$.

This parameter determines how strongly differences affect changes in connectivities between agents. For the sake of comparison we briefly summarize notable aspects of the solutions in this case. The value of this parameter is not important for any particular scenario, as the other numerical values were chosen merely to illustrate the model dynamics. Rather, the parameter value of $b$ is reported here as a means of tracking the effects on clustering behavior with each further modification.

In the deterministic case without delay, the characteristic values appear to change monotonically before clusters are formed, and only slight variations from this are observed with the introduction of stochasticity. Even when stochasticity is included, clusters do not break apart unless the noise dominates the outcome and network structure collapses entirely. Furthermore, in the original results of 8 and in the reproduction of these results, sufficiently small amounts of stochasticity do not affect the number of clusters formed when compared to the deterministic case.

4.1. Delayed deterministic social network model. We considered the deterministic system $((\sigma, \gamma)=(0,0))$ with varying parameters $b$ and $\tau$ on longer time intervals $T=50$ to better quantify long-term behavior. The three-cluster scenarios (in which the agents do not affect each other's characteristics and therefore remain in the same original clusters as in the initial conditions) appear to be robust in the presence of delays. Even large delays do not seem to affect the solutions of the model in this parameter regime (not shown).

In contrast, for parameter values that produce a one-cluster scenario (depicted in Figure 11), the characteristic values of the agents are affected as seen in Figure 2. The characteristics of Agents 2 and 7, which are initially different from those of the other agents, oscillate depending on the size of the delay before joining the cluster of other agents. That is, the trajectories oscillate less with smaller delays, and the amplitude of the oscillations increases with increasing size of delay. All agents other than Agent 2 and Agent 7 behave identically and so are represented in these depictions as Agent 1.

Although the top panels (a) and (b) of Figure 2 depict the characteristics of Agents 2 and 7 as being significantly different than those of the other agents at the peaks of 


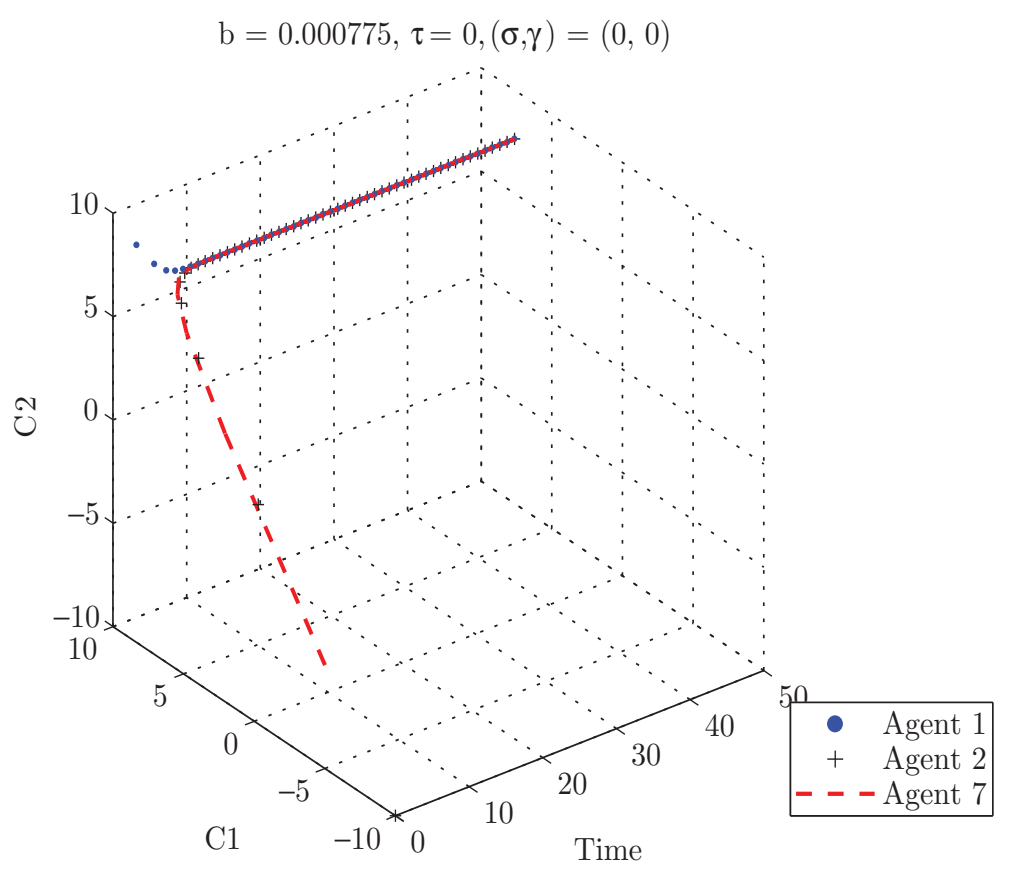

FIG. 1. One-cluster $(b=0.00075)$ scenario without delay.

the oscillations, the connectivities shown in panels 2(c) and 2(d) are positive for all agents. Thus, this scenario is interpreted as a one-cluster outcome. In general, when the characteristics are oscillating around each other and do not diverge over a substantial time interval, the connectivities between those agents are positive and they are in the same cluster.

While the long-term behavior of the one- and three-cluster scenarios is observed regardless of the size of delay, the two-cluster outcome is not observed with a sufficiently large delay. In the absence of any delay (with or without stochasticity), two clusters were formed for $b \in[0.000155,0.01390)$. Agents 2 and 7 join one cluster and all the other agents form the other (Figure 3). With large delays (Figure 4(c)], the oscillations in the characteristics of Agents 2 and 7 grow until they join the cluster of the other agents (represented as Agent 1). For smaller delays $(\tau=0.5$ in Figure 4(a), characteristics oscillate, but the original two-cluster outcome results. As the delay is increased, the oscillations increase in magnitude and in the time interval during which they are observed. It appears that the delay has a 'destabilizing' effect for the two-cluster scenario such that the oscillations continue once a threshold value of $\tau$ is reached (Figure 4(b)). For delays larger than a threshold value, the oscillations grow such that the two-cluster scenario grows into a single cluster of all agents (Figure 4(c)) . Delays have this effect for smaller values of $b$ in the interval $[0.000155,0.01390)$. Connectivities are shown in the bottom panels of Figure 4 for $b=0.002$ to show the cluster formation and dissolution for the characteristics in the corresponding panels along the top row. 


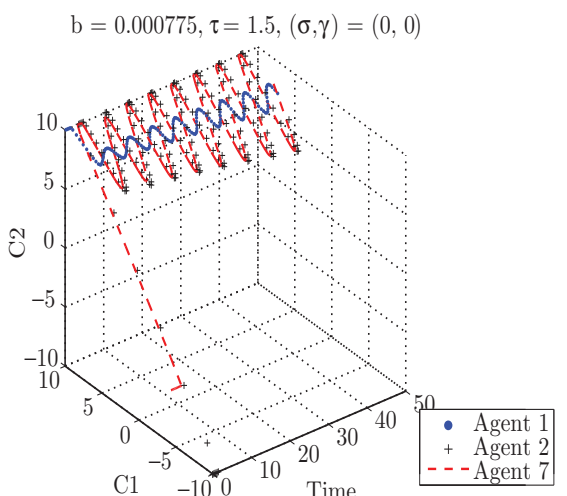

(a) Agent characteristics with delay $\tau=1.5$

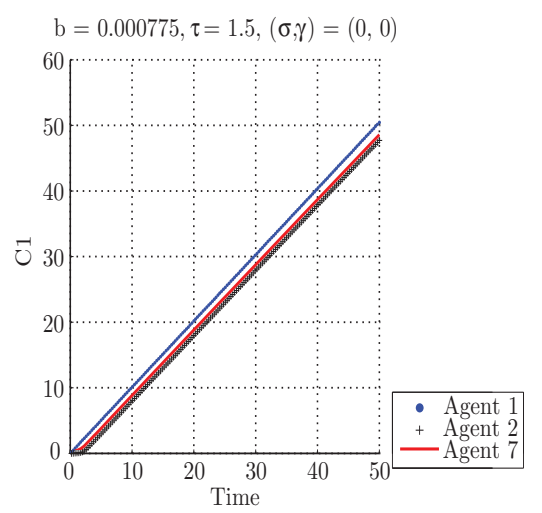

(c) Connections of agent 1 to other agents with delay $\tau=1.5$

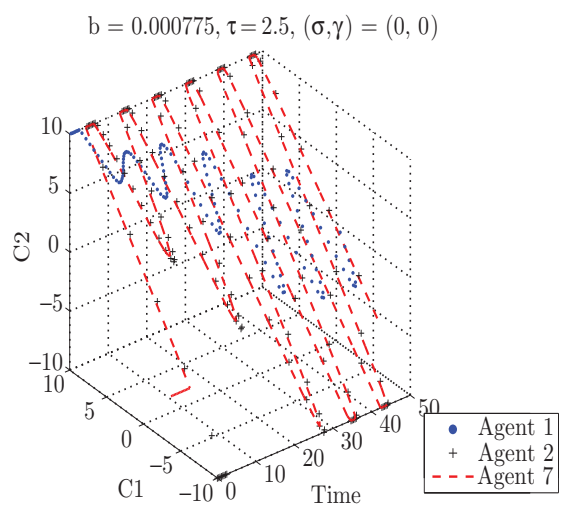

(b) Agent characteristics with delay $\tau=2.5$

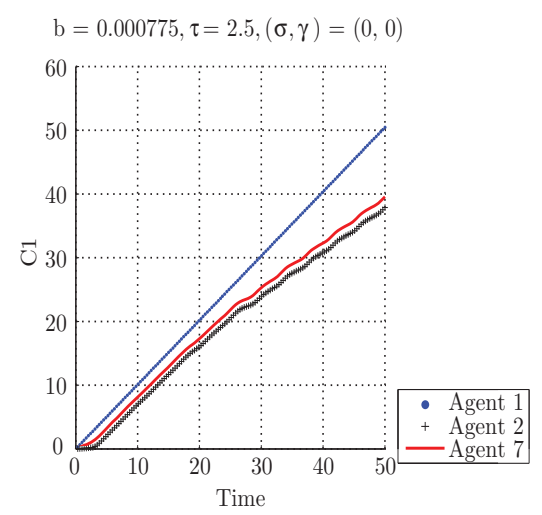

(d) Connection of agent 1 to other agents with delay $\tau=2.5$

FIG. 2. One-cluster $(b=0.00075)$ outcomes with the addition of large delays $(\tau=1.5,2.5)$.

Similarly, as seen in Figure 5, the delay has a destabilizing effect for the other values of the parameter $b$ that produce a two-cluster scenario (larger values of $b \in(0.002,0.01390)$ ). For smaller values of delay such as $\tau=0.5$, oscillations appear for a short time interval and again dampen so that the two-cluster scenario is preserved. If the delay is increased slightly to $\tau=0.75$, it reaches what appears to be a threshold where the oscillations are sustained (Figure 5(b) . Once the delay is increased further, the two-cluster scenario forms, although oscillations grow drastically in magnitude. A three-cluster scenario is briefly established $(t \approx 22$ in Figure $5(\mathrm{c}))$, and the oscillations resume as Agents 2 and 7 form the second cluster again. For larger delays, the three-cluster outcome is sustained eventually (see Figure 5(d) , and the second cluster does not re-form. The characteristics of Agents 2 and 7 become less alike with each brief three-cluster configuration, and eventually, they are sufficiently distinct to not influence each other again. 


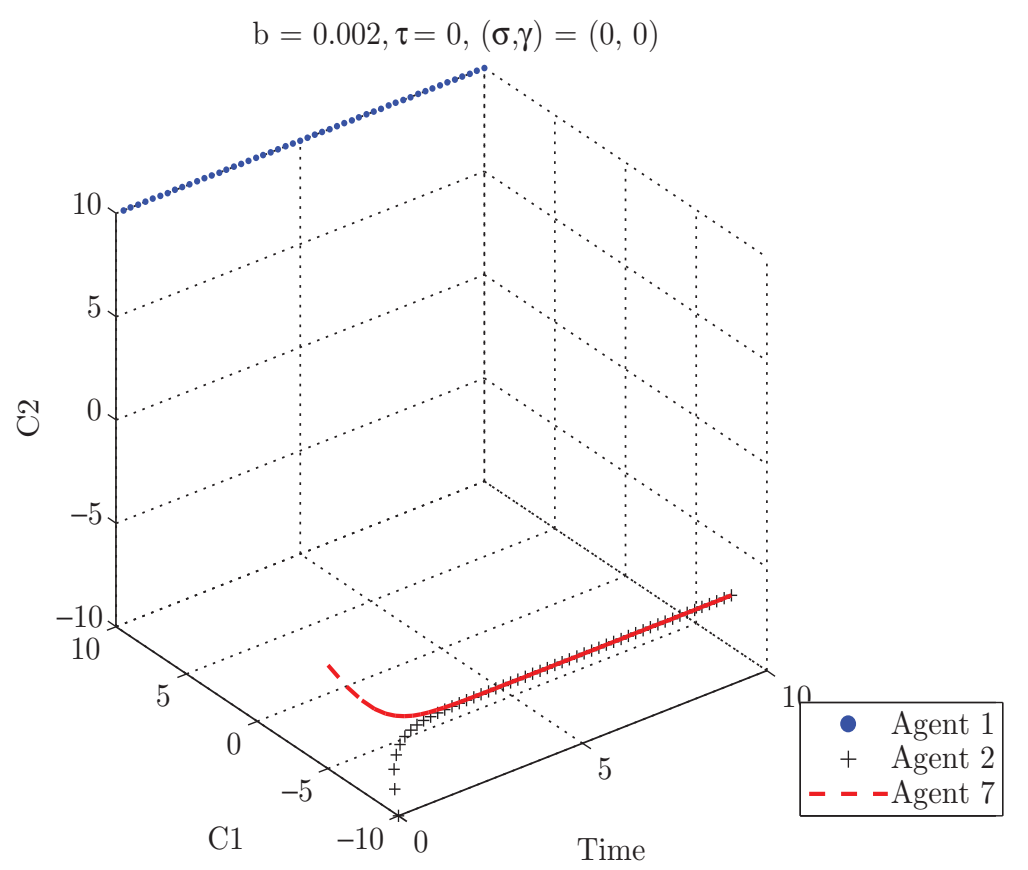

FIG. 3. Two-cluster $(b=0.002)$ scenario without delay.

Of notable significance, we observed a novel clustering outcome different from those previously observed, shown in Figure 6 with $b=0.0135$ and $\tau=1.3$. A new twocluster scenario is formed when characteristics oscillate greatly until one of either Agent 2 or 7 joins the cluster of all other agents (represented by Agent 1). In simulations, the differences between the frequencies with which Agent 2 or 7 was observed to join the cluster of other agents was not noticeable. So this configuration is termed $2 B$ throughout the remainder of this paper.

From these results, one could not conclude that small delays, which may be more realistic, do not matter in the deterministic social network model. Small delays were shown to affect characteristic dynamics even though the long-term behavior was not affected (particularly in the original two-cluster scenario). Incorporating stochasticity, which represents another aspect of physical realism that one cannot reasonably ignore, could exacerbate these oscillations in unknown ways.

4.2. Delayed stochastic social network model. We first note that extensive stochastic realizations were run in 8 for only one value of $b$ - one that produced a 1-cluster scenario. Because the behavior of the model was significantly affected for other parameter values, we ran many solutions (not all depicted here) without delay. We use these solutions as a means to illustrate which changes in solution behavior were due to delay and which were due to stochasticity in the delayed system.

We discuss results for the case without delay $(\tau=0)$, 'small' delay $(\tau=0.75)$, and 'large' delay $(\tau=1.3)$ specifically. The effects of stochasticity were observed at 


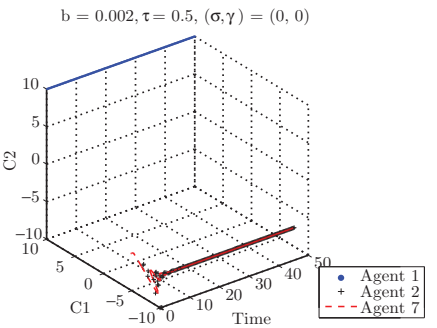

(a) Characteristics with small delay $\tau=0.5$

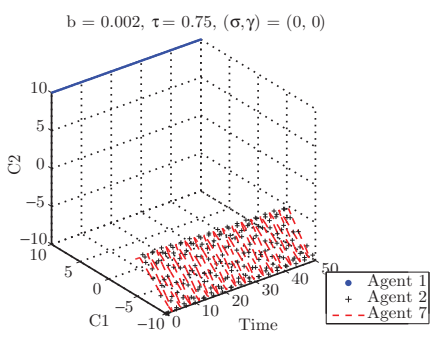

(b) Characteristics with medium delay $\tau=0.75$

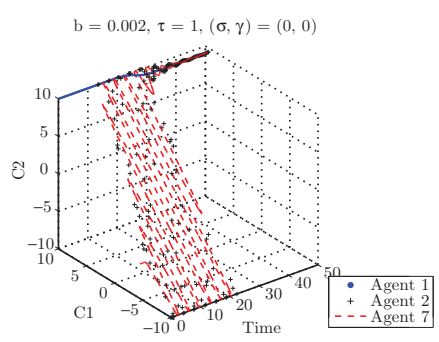

(c) Characteristics with large delay $\tau=1.0$

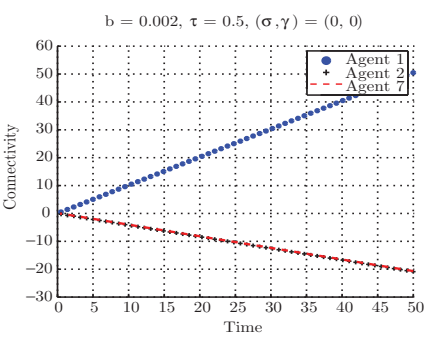

(d) Connectivities to Agent 1 with small delay $\tau=0.5$

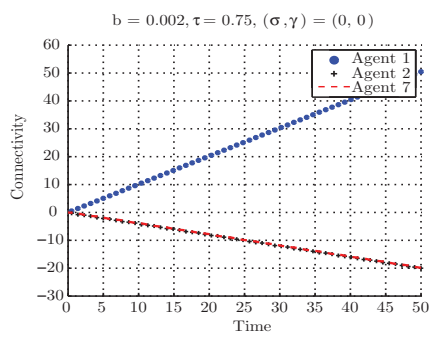

(e) Connectivities to Agent 1 with medium delay $\tau=0.75$

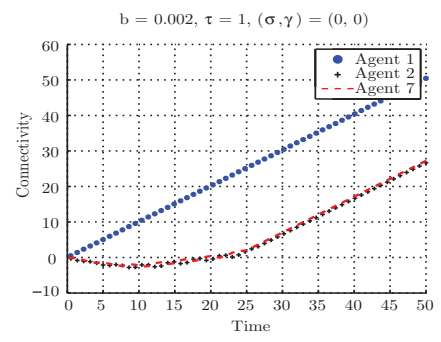

(f) Connectivities to Agent 1 with large delay $\tau=1.0$

FIG. 4. Outcomes of simulations using $b=0.002$ (which produces a two-cluster scenario for $\tau=0)$ with small $(\tau=0.5)$, medium $(\tau=0.75)$, and large $(\tau=1.0)$ delay. 


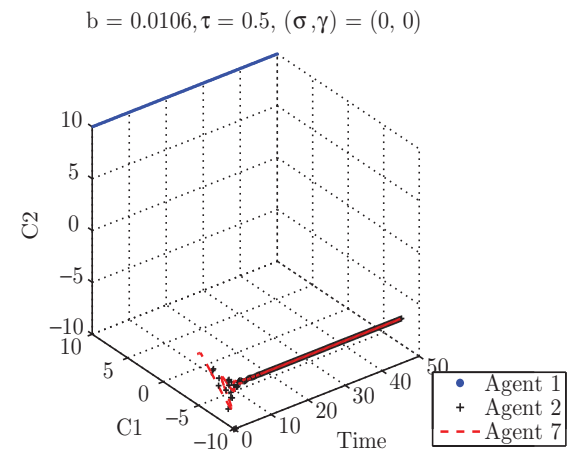

(a) Characteristics with small delay $\tau=0.5$

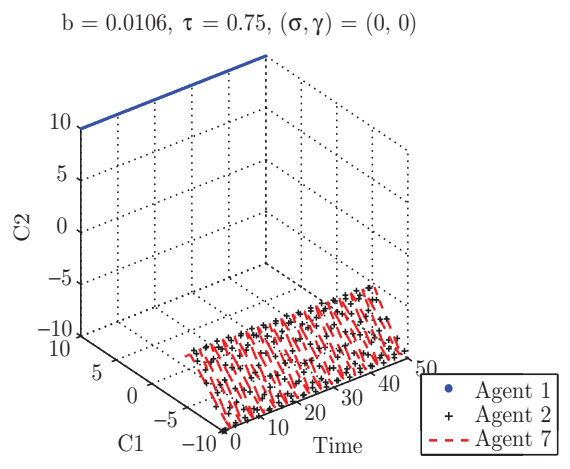

(b) Characteristics with 'threshold' delay $\tau=0.75$

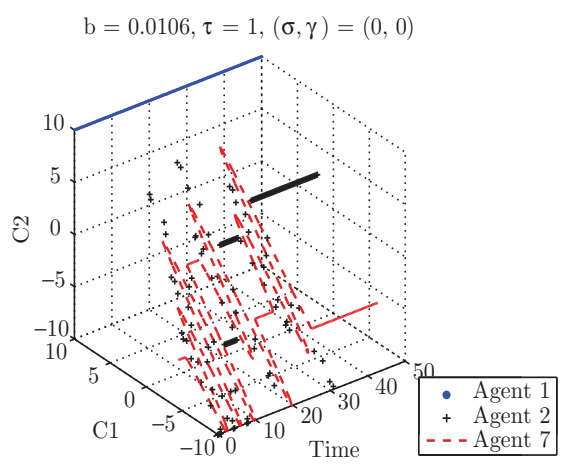

(d) Characteristics with large delay $\tau=1.0$ (c) Characteristics with 'above threshold'

delay $\tau=0.85$

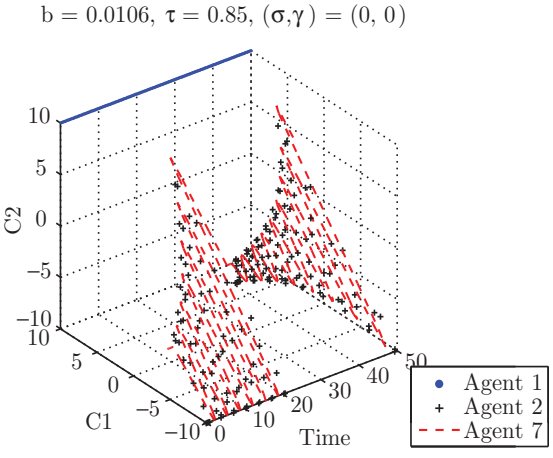

Fig. 5. Outcomes of simulations using $b=0.0106$ (which produces a two-cluster scenario for $\tau=0$ ) for $\tau=0.5,0.75,0.85,1.0$.

the following levels of stochasticity $(\sigma, \gamma):(1,0.5),(2,1),(5,6)$. At high levels of noise, $(\sigma, \gamma)=(5,6)$, all agents joined one cluster with their characteristics oscillating. Since this behavior was observed in all cases, these solutions are neither shown here nor discussed further.

The outcome of all agents forming one cluster is robust to the addition of both stochastic noise and delay. Both of these effects result in the introduction of oscillations in characteristics, and together the effects are additive. These solutions are not shown as they do not differ qualitatively from the stochastic studies in [8]. That is, with increasing noise and/or delay size, the magnitude of the oscillations also increases. However, the agents remain in the same cluster (all connectivities remain positive) unless the noise dominates the dynamics and the network structure is lost. 


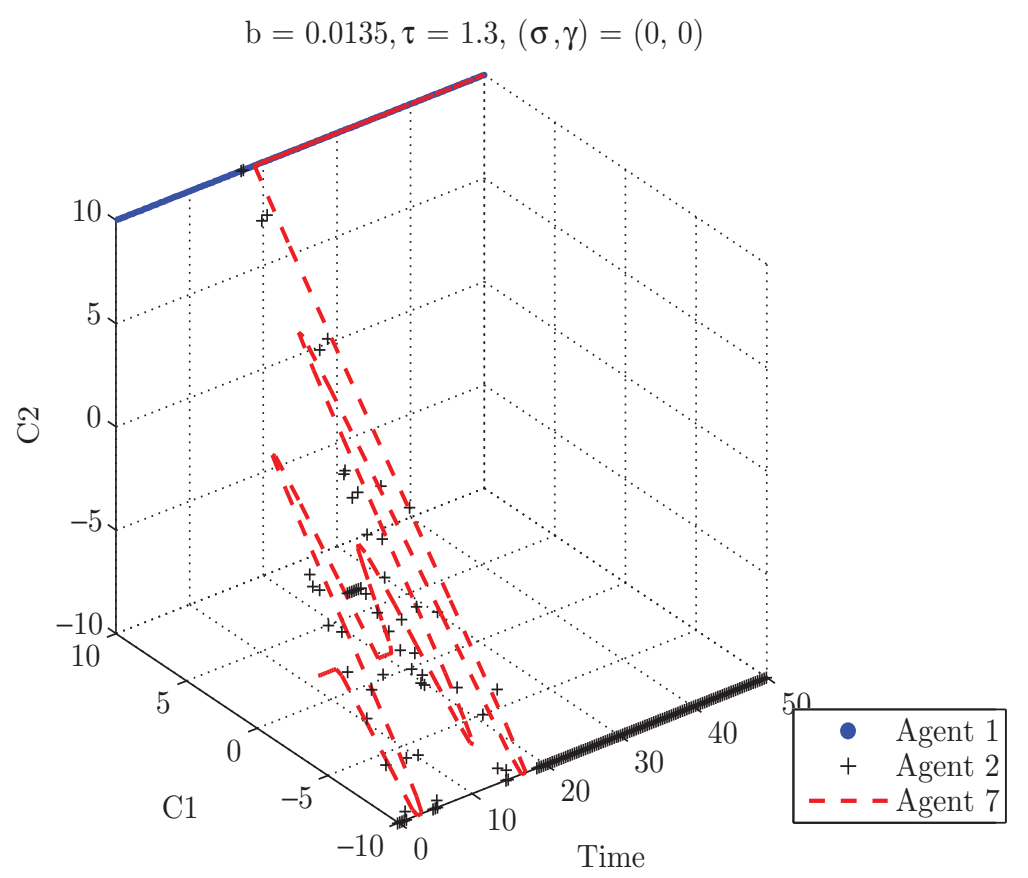

Fig. 6. Original two-cluster $(b=0.0135)$ scenario with delay $\tau=1.3$ giving rise to a novel $2 B$ scenario.

When $b$ is increased to the lower portion of the original 2-cluster interval $[0.00155,0.01390)$, stochasticity in the non-delayed case can result in all the agents joining the same cluster in roughly half of the observed realizations, as shown in Table 1 , This was not reported as observed in the study 8 , where it would have been termed a "noise-enlarged" scenario. Recall that large delays in this parameter range (Figure 4) also resulted in one cluster forming. Increasing noise results in small irregular characteristic oscillations ((a) and (b) of Figure 7), while the addition of noise to the delayed model ((c) and (d) of Figure 7) dampen the larger and more regular oscillations seen in the deterministic case. As the delay and hence the oscillations increase, Agents 2 and 7 join the cluster of the other agents regardless of the level of uncertainty considered. Stochasticity and delay are seen to have the same effect on the solution, transforming a two-cluster scenario to a one-cluster scenario, and each modification induces oscillations when considered separately. However, in a sense these aspects tend to cancel each other out when taken together as the stochasticity destabilizes the oscillations induced by the delay.

For the larger values of $b$ in the original 2-cluster scenario range, the delays were seen to destabilize the 2-cluster into a 3-cluster outcome (Figure 5). When stochasticity is incorporated, a wide range of outcomes are observed. Most notably, one of either Agent 2 or 7 joins the cluster of other agents (represented by Agent 1 in the figures), and the other agent (either 7 or 2) remains solitary. An example of this (labeled as scenario $2 B$ ) is shown in Figures 8(b) and 8(e). From the computations carried out in this study, it 


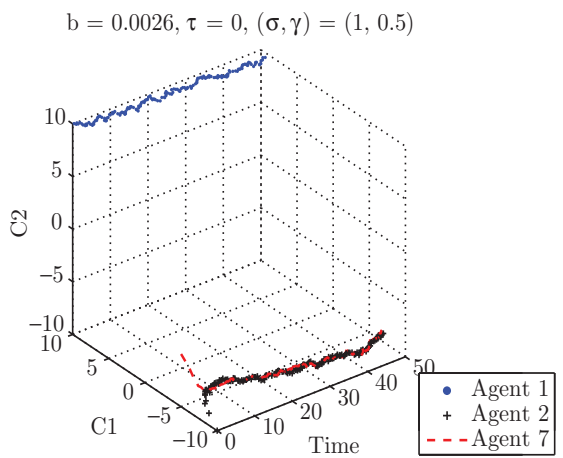

(a) $\tau=0,(\sigma, \gamma)=(1,0.5)$

$\mathrm{b}=0.0026, \tau=0.75,(\sigma, \gamma)=(1,0.5)$

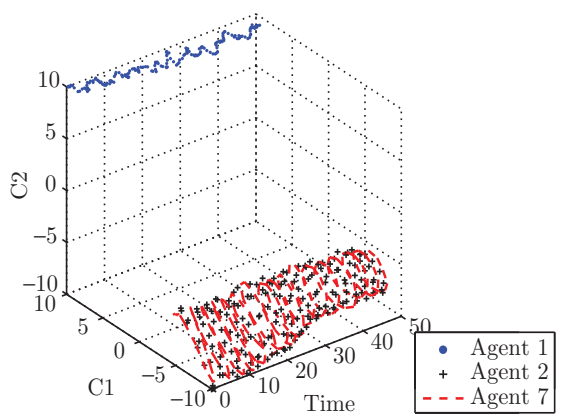

(c) $\tau=0.75,(\sigma, \gamma)=(1,0.5)$

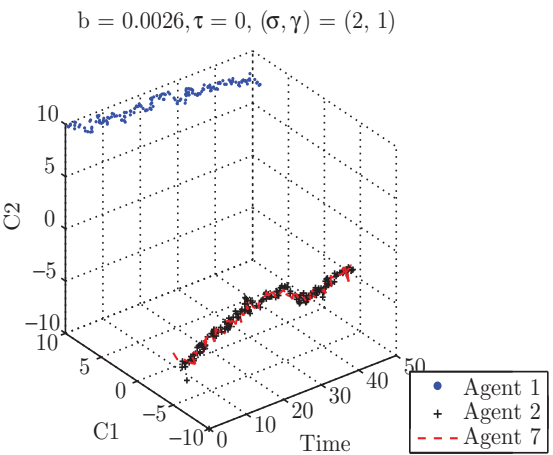

(b) $\tau=0,(\sigma, \gamma)=(2,1)$

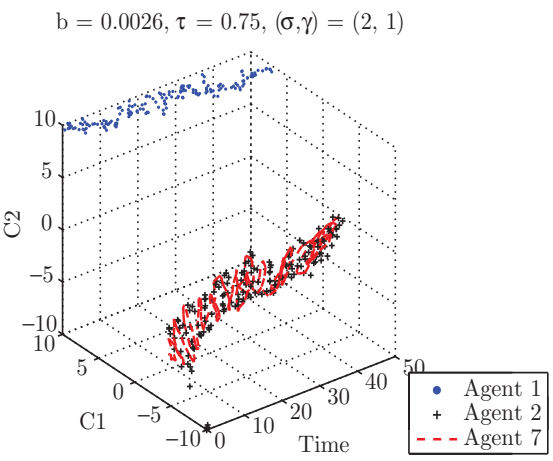

(d) $\tau=0.75,(\sigma, \gamma)=(2,1)$

FIG. 7. Characteristics for typical two-cluster outcomes when $b=$ 0.0026 for low $(\sigma, \gamma)=(1,0.5)$ and medium $(\sigma, \gamma)=(2,1)$ noise (columns) and no $(\tau=0)$ and small $(\tau=0.75)$ delay (rows).

does not appear that one of Agents 2 or 7 joins the main cluster more frequently than the other, so they are considered equivalent outcomes. This is a configuration not observed in the non-delayed case and supports motivation to consider such aspects in network applications as they might well be representative of actual observed scenarios.

Again, it appears that the addition of moderate levels of stochasticity restores the solutions to more closely resemble their non-delayed deterministic trajectory. In this case, the oscillations that lead to a 3-cluster scenario are exacerbated, rather than dampened, by stochasticity, leading to the wide range of outcomes shown in Figure 8 . Further, the frequency with which the 3 -cluster scenario is observed decreases with increasing noise levels (Table 2). However, a considerable proportion of the outcomes are not observed in delayed, deterministic, or stochastic versions of the model. Therefore, simulation results of a network model neglecting to incorporate delayed and/or stochastic effects in a system where they are likely present may be questionable. 


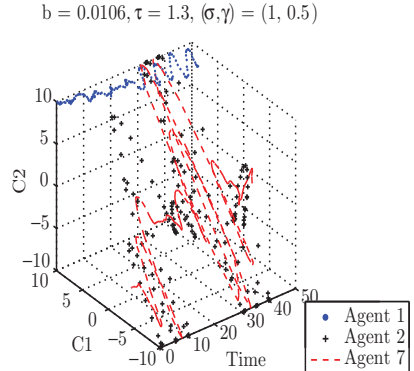

(a) 2 cluster characteristics, $(\sigma, \gamma)=(1,0.5)$

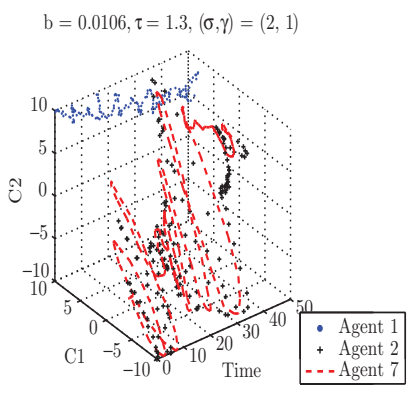

(d) 2 cluster characteristics, $(\sigma, \gamma)=(2,1)$
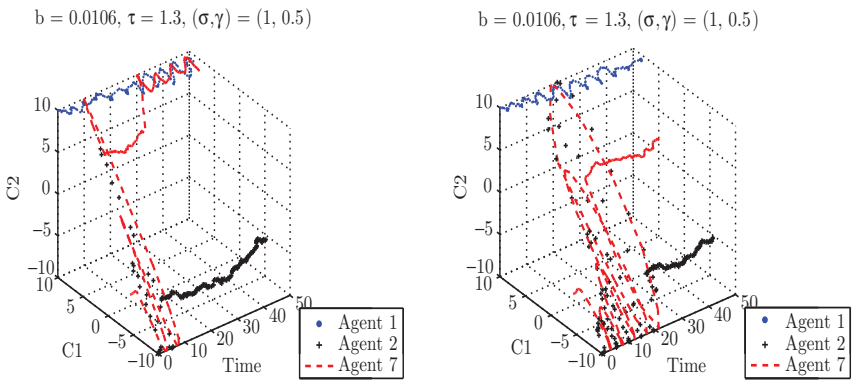

(b) $2 B$ cluster characteristics, $(\sigma, \gamma)=(1,0.5)$

(c) 3 cluster characteristics, $(\sigma, \gamma)=(1,0.5)$

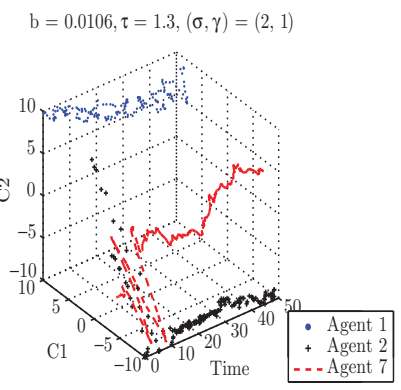

(e) $2 B$ cluster characteristics, $(\sigma, \gamma)=(2,1)$ (f) 3 cluster characteristics, $(\sigma, \gamma)=(2,1)$

FIG. 8. Characteristics for typical outcomes when $b=0.0106$ and delay $\tau=1.3$ for low $(\sigma, \gamma)=(1,0.5)$ and medium $(\sigma, \gamma)=(2,1)$ noise. The frequencies with which each outcome was observed is reported in Table 2 
TABLE 1. Clustering outcomes for $b=0.0026$ without delay $(\tau=0)$, and with small $(\tau=0.75)$ and large $(\tau=1.3)$ delay, and for varying levels of stochasticity $(\sigma, \gamma)=(0,0),(\sigma, \gamma)=(1,0.5),(\sigma, \gamma)=(2,1)$. Numbers given are for the fraction out of total number of simulations of occurrences of the behavior observed.

\begin{tabular}{|c|c|c|c|}
\hline$\tau$ & $(\sigma, \gamma)$ & 1 cluster & 2 cluster \\
\hline 0 & $(0,0)$ & & 1 \\
& $(1,0.5)$ & 0.01 & 0.99 \\
& $(2,1)$ & 0.47 & 0.53 \\
\hline 0.75 & $(0,0)$ & & 1 \\
& $(1,0.5)$ & & 1 \\
& $(2,1)$ & 0.23 & 0.77 \\
\hline 1.3 & $(0,0)$ & & 1 \\
& $(1,0.5)$ & & 1 \\
& $(2,1)$ & & 1 \\
\hline
\end{tabular}

TABLE 2. Clustering outcomes for $b=0.0106$ with large delay $(\tau=$ $1.3)$, and with varying levels of stochasticity $(\sigma, \gamma)=(0,0),(\sigma, \gamma)=$ $(1,0.5),(\sigma, \gamma)=(2,1)$. Numbers given are for the fraction out of 100 simulations of occurrences of the behavior observed.

\begin{tabular}{|c|c|c|c|c|}
\hline$(\sigma, \gamma)$ & 1 cluster & 2 cluster & 2B cluster & 3 cluster \\
\hline$(0,0)$ & & & & 1 \\
\hline$(1,0.5)$ & 0.01 & 0.13 & 0.34 & 0.52 \\
\hline$(2,1)$ & 0.04 & 0.33 & 0.37 & 0.26 \\
\hline
\end{tabular}

5. Concluding remarks. Because agent clustering is one of the more important features of dynamic networks, it is of great value to understand the stability of clustering behavior under perturbations such as noise (stochasticities) in the dynamics or delays in characteristic responses to contacts or information. As reported here, by considering either delays or stochastic effects, one can obtain profoundly different outcomes with respect to agent clustering in the network models proposed in this paper. Even when the long-term outcome is unchanged with the addition of small delays, there are changes in short-term dynamics of the solution that are exacerbated by the addition of a small amount of noise. These findings suggest that it is important to consider both delays and stochasticity if they may be relevant to the network application.

Through the extensive computations performed in this study, we have identified richer behavior than reported in 8 when uncertainty is incorporated into the model even in the absence of a delay. Cluster switching (e.g., a 2-cluster scenario ending up as a 1cluster scenario) was observed with the addition of stochasticity that was not seen in [8], which would have been called "noise-enlarged" in that effort. However, uncertainty incorporated in the network model via a system of stochastic differential equations represents only one way to improve upon the physical, social, or biological fidelity of the 
mathematical representation, and novel behavior is observed when the model is further extended to include delays.

When the delay is considered in the deterministic version of the model, an outcome that was commonly observed in the non-delayed case is not observed at all for sufficiently large delays. Even smaller delays result in clusters that can be broken by the addition of a small amount of stochasticity. Further, including stochasticity along with delays produces results in cluster forming (the $2 B$ scenarios) that are not observed otherwise.

These studies support the notion that neither delays nor stochasticity can be neglected in models when present in a social network application if a reasonably realistic representation of the social network is desired. Networks incorporating both stochasticity and delay are of interest in a wide range of applications, and an accessible framework to study such systems is of considerable interest to many. Here we have demonstrated that a dynamical system as opposed to computer-based nodal networks is an amenable framework in which to study these effects in the context of a network.

Acknowledgements. This research was supported in part by the National Institute of Allergy and Infectious Diseases under Grant Number R01AI071915-07 and in part by the Air Force Office of Scientific Research under grant number FA9550-09-1-0226.

\section{REFERENCES}

[1] A. V. Balakrishnan, Active control of airfoils in unsteady aerodynamics, Appl. Math. Opt., 4 (1978), 171-195. MR0468596 (57:8428)

[2] H. T. Banks, Control of functional differential equations with function space boundary conditions, in Delay and Functional Differential Equations and Their Applications (K. Schmitt, ed.), Academic Press, New York, 1972, 1-16. MR0389319 (52:10150)

[3] H. T. Banks, Parameter identification techniques for physiological control sysems, in Mathematical Aspects of Physiology (F. C. Hoppensteadt, ed.), Amer. Math. Soc., Providence, 1981, 361-383. MR.623301 (82k:92020)

[4] H. T. Banks, Identification of nonlinear delay systems using spline methods, in Nonlinear Phenomena in Mathematical Sciences (V. Lakshmikantham, ed.), Academic Press, New York, 1982, $47-55$.

[5] H. T. Banks, D. M. Bortz and S. E. Holte, Incorporation of variability into the mathematical modeling of viral delays in HIV infection dynamics, Math. Biosciences, 183 (2003), 63-91. MR1965457 (2004b:92032)

[6] H. T. Banks and J. A. Burns, Hereditary control problems: Numerical methods based on averaging approximations, SIAM J. Control and Optimization, 16 (1978), 169-208. MR0483428 (58:3430)

[7] H. T. Banks, J. A. Burns and E. M. Cliff, Parameter estimation and identification for systems with delays, SIAM J. Control and Optimization, 19 (1981), 791-828. MR634954(84e:93050)

[8] H. T. Banks, A. F. Karr, H. K. Nguyen and J. R. Samuels, Jr., Sensitivity to noise variance in a social network dynamics model, Quarterly Applied Math., 66 (2008), 233-247. MR2416772 (2009b:91123)

[9] H. T. Banks and F. Kappel, Spline approximations for functional differential equations, J. Differential Equations, 34 (1979), 496-522. MR.555324 (81c:65031)

[10] H. T. Banks, K. L. Rehm and K. L. Sutton, Conversion of a dynamic social network stochastic differential equation model to Fokker-Planck model, Technical Report CRSC-TR09-10, Center for Research in Scientific Computation, North Carolina State University, April, 2009.

[11] R. Bellman and K. L. Cooke, Differential Difference Equations, Academic Press, New York, 1963. MR0147745 (26:5259)

[12] S. Blythe, X. Mao and X. Liao, Stability of stochastic delay neural networks, J. Franklin Institute, 338 (2001), 481-495. MR.1833972 (2002c:93173)

[13] E. Boukas and Z. Liu, Deterministic and Stochastic Time Delay Systems, Springer-Verlag, New York NY, 2002. 
[14] P. J. Carrington, J. Scott and S. Wasserman, Models and Methods in Social Network Analysis, Cambridge University Press, New York, 2005.

[15] R. D. Driver, Some harmless delays, in Delay and Functional Differential Equations and Their Applications (K. Schmitt, ed.) Academic Press, New York, 1972, 103-119. MR0385277 (52:6141)

[16] L. E. El'sgol'ts, Introduction to the Theory of Differential Equations with Deviating Arguments, Holden-Day, San Francisco, 1966. MR0192154 (33:381)

[17] G. R. Grummett and D. R. Stirzaker, Probability and Random Processes, $2^{\text {nd }}$ edition, Oxford University Press, Oxford, UK, 1992. MR1199812 (93m:60002)

[18] A. Halanay, Differential Equations: Stability, Oscillations, Time Lags, Academic Press, New York, 1966. MR 0216103 (35:6938)

[19] J. K. Hale, Functional Differential Equations, Appl. Math. Sci., Vol. 3, Springer-Verlag, New York, 1971. MR0466837 (57:6711)

[20] Y. Jiang and Y. Liu, Stochastic Network Calculus, Springer-Verlag, New York NY, 2008.

[21] N. Minorsky, Experiments with activated tanks, Trans. ASME, 69 (1941), 735-747.

[22] N. Minorsky, Self-excited oscillations in dynamical systems possessing retarded actions, J. Appl. Mechanics, 9 (1942), A65-A71.

[23] N. Minorsky, Nonlinear Oscillations, Van Nostrand, New York, 1962. MR0137891 (25:1339)

[24] K. Park and W. Willinger, Self-similar Network Traffic and Performance Evaluation, John Wiley \& Sons, Inc., New York NY, 2000.

[25] S. Wasserman and K. Faust, Social Network Analysis: Methods and Applications, Cambridge University Press, New York, 1994.

[26] S. Wasserman and J. Galaskiewicz, Advances in Social Network Analysis: Research in the Social and Behavioral Sciences, Sage Publications, Thousand Oaks, CA, 1994. 